\title{
Autorizzativo e autorizzatorio
}

\section{Vittorio Coletti}

PUBBLICATO: 17 APRILE 2020

\section{Quesito:}

Alcuni lettori ci sottopongono gli aggettivi autorizzativo e autorizzatorio in uso nei testi normativi (si parla ad esempio di regime autorizzatorio): il loro impiego è "corretto"? Sono equivalenti o è meglio usare uno dei due?

\section{Autorizzativo e autorizzatorio}

Autorizzatorio e autorizzativo sono due aggettivi derivati dal verbo autorizzare, l'uno con suffisso -orio e l'altro con suffisso -ivo, due affissi che secondo lo Zingarelli 2019 producono rispettivamente oltre 370 e oltre 930 aggettivi, spesso deverbali come quelli qui in esame. Dei due solo autorizzativo ha avuto, sin qui, un po' di ospitalità nei dizionari generali a stampa. Ad esempio, nel Supplemento 2009 al GDLI, dove si legge:

\begin{abstract}
Autorizzativo, agg. Burocr. Che si riferisce alle autorizzazioni; che serve a concedere un'autorizzazione. La Repubblica [20-X-1984], 44: L'Enel insiste nel chiedere uno snellimento dell'iter autorizzativo delle centrali elettriche. Corriere della Sera [30-IX-2003]: Si può dire casomai che ai tempi del monopolio la programmazione di fabbisogni e investimenti, facendo capo a un unico soggetto, era più facile e anche i processi autorizzativi erano meno complessi di quelli attuali.
\end{abstract}

$=$ Agg, verb. da autorizzare

O nel Devoto-Oli 2ori, che lo definisce come "relativo alla concessione o finalizzato all'ottenimento di un'autorizzazione: potere a.; iter, procedimento a.", datandolo alla prima metà del XX secolo o nello Zingarelli 2019 che lo registra e data al I98I con esempi come provvedimento e iter autorizzativo. Lo registrano anche i Neologismi della Treccani in rete con una citazione da "Repubblica" del 2005, in cui si parla di "processo autorizzativo" e il Reverso italiano inglese online con non pochi esempi. Lo riporta pure il dizionario online multilingue Glosbe, che ne attesta molte occorrenze.

Lo stesso dizionario registra anche alcune (poche) testimonianze di autorizzatorio, quasi tutte da fonti del diritto, come in:

Il Comitato ritiene che sia necessario escludere dal novero dei rifiuti, e quindi dal relativo regime autorizzatorio, le apparecchiature elettriche ed elettroniche - selezionate mediante semplici operazioni di cernita nei centri di raccolta - e successivamente destinate al reimpiego anche previa usuale riparazione.

Autorizzatorio è registrato infine pure dai Neologismi Treccani 2018 con un esempio del 20I2. Va detto che se i dizionari comuni sembrano essere stati restii ad ospitare i due aggettivi, questi sono invece entrambi ben presenti nel lessico giuridico-amministrativo corrente e a volte entrano in lessemi complessi come potere autorizzatorio ("il potere attribuito alla pubblica amministrazione di rimuovere con un provvedimento [detto appunto provvedimento autorizzatorio o autorizzazione] i limiti posti dall'ordinamento all'esercizio di una preesistente situazione giuridica soggettiva di vantaggio", Wikipedia) e l'analogo potere autorizzativo (Salvatore Berlingò, Il potere autorizzativo nel diritto 
ecclesiastico, Giuffrè, 1974).

Il significato dei due aggettivi ("che rilascia, è volto a rilasciare un'autorizzazione") è sostanzialmente lo stesso, come si vede. Spesso si usa l'uno o l'altro, senza particolare differenza, come in questo provvedimento ufficiale riportato su "Ipsoa, Quotidiano premium" del is/6/2019 (anche se il plurale -tori potrebbe risalire pure a un singolare agentivo in -tore che è già trecentesco, come dal TLIO; il GDLI lo cita, sotto altra voce, dal Dizionario amministrativo del Rezasco I88I, il quale invece non registra né autorizzatorio né autorizzativo):

Nella disciplina posta dalla Regione autonoma Valle d'Aosta, il provvedimento di VIA è autonomo rispetto agli altri atti autorizzatori connessi alla realizzazione dell'opera, in evidente deroga all'assetto unitario e onnicomprensivo del provvedimento unico previsto dal codice dell'ambiente. La sentenza della Corte Costituzionale n. I47 del I9 giugno 2019 entra nel merito degli articoli ro, I2 e I3 della legge regionale della Valle d'Aosta n. 3 del 2018 censurati dall'Avvocatura generale dello Stato in quanto configurerebbero il provvedimento di VIA (Valutazione Impatto Ambientale) regionale, quale atto autonomo da integrare nell'atto autorizzativo rilasciato da altre strutture regionali competenti.

O in questo brano da F. Nesta, Brevi cenni sul procedimento amministrativo, Umbrialex.it:

[...] nel quadro della semplificazione amministrativa è prevista all'art. Ig la possibilità per i privati di intraprendere l'esercizio di un'attività sulla base di una mera denuncia - dichiarazione sostitutiva senza dover conseguire il prescritto titolo autorizzativo [...] Altra figura importante, nell'ottica della liberalizzazione dell'attività dei privati e di miglioramento del rapporto tra P.A. e cittadini, è quella del silenzio-assenso (art. 2o), secondo la quale la stessa P.A. deve individuare i casi in cui la domanda di rilascio di un provvedimento autorizzatorio - al fine di esercitare un'attività privata - si considera accolta qualora non venga comunicato all'interessato il provvedimento di diniego entro un dato termine...

La ricerca su Google mostra che provvedimento autorizzativo ricorre più di 80 mila volte e provvedimento autorizzatorio circa la metà (comunque tanto) e che, al contrario, procedimento autorizzatorio prevale con oltre 37 mila attestazioni sul per altro molto solido (oltre 29 mila) procedimento autorizzativo. Atto autorizzativo è (forse anche per la minor cacofonia?) molto più numeroso (oltre 67 mila) di atto autorizzatorio (comunque non scarso: oltre 13 mila). Insomma, due buoni contribuenti del lessico speciale giuridico.

A conferma delle ragioni di miglior accoglienza in qualche dizionario della lingua comune, autorizzativo sembra preferito a autorizzatorio (iter autorizzativo è su Google oltre I40 mila volte, iter autorizzatorio solo 6.500), che il mio correttore di Google considera erroneo, anche se il regime autorizzatorio di cui parla una lettrice supera di gran lunga (oltre 40 mila attestazioni su Google) quello autorizzativo (comunque alla quota rispettabile di quasi 20 mila).

Va detto che queste due tipologie di aggettivi sono assai diffuse nel linguaggio giuridico, sia quella in -torio (assolutorio, accusatorio, commissorio, promissorio ecc.) che quella in -ivo (commissivo, detentivo, impugnativo). In genere (come è ovvio), i corradicali non sono sinonimi, ma qualche volta, sia pure con diverso grado di fortuna, lo sono, sia nella speciale lingua giuridica che in quella colta generale (assertorio e assertivo, collusorio e collusivo, compilatorio e compilativo, diffamatorio e diffamativo, emendatorio e emendativo, esecutorio e esecutivo, illusorio e illusivo, suppletoria e suppletiva ecc). Dunque niente di strano che lo siano anche autorizzatorio e autorizzativo, dei quali, piacciano o no, i dizionari dell'uso dovrebbero ormai prendere atto. Tanto più che non si possono neppur più dire dei neologismi. Google libri ci attesta autorizzatorio (provvedimento autorizzatorio) sulla "Rivista 
trimestrale di diritto pubblico" del 1965 e autorizzativo (decreto autorizzativo) addirittura già nel I835 ("Gazzetta di Firenze", Leggi).

Dunque, due sinonimi corretti e ammissibili, da inserire nei lemmari dell'italiano. Sarebbe auspicabile una loro specializzazione in collocazioni diverse: gli esempi appena forniti ce la fanno già intravedere e non è detto che in futuro non succeda più regolarmente.

\section{Cita come:}

Vittorio Coletti, Autorizzativo e autorizzatorio , "Italiano digitale", 2019, XIII, 2020/2 (aprile-giugno) DOI: $10.35948 / 2532-9006 / 2020.3306$

\section{Copyright 2019 Accademia della Crusca}

Pubblicato con licenza creative commons CC BY-NC-ND 